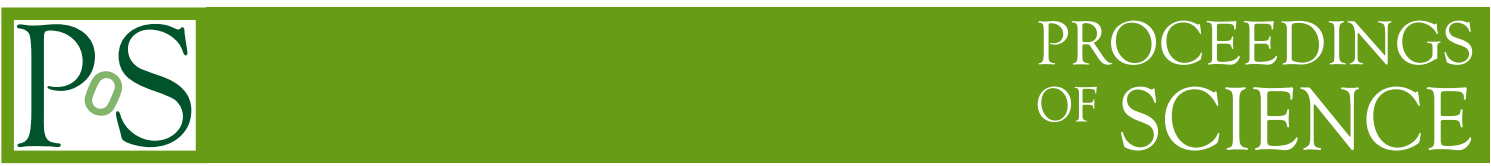

\title{
The KM3NeT project: status and future perspectives
}

\author{
Carla Distefano* for the KM3NeT Collaboration \\ Laboratori Nazionali del Sud, INFN, Catania, Italy \\ E-mail: distefano_c@lns.infn.it
}

\begin{abstract}
The KM3NeT Collaboration is constructing a multi-site research infrastructure in the Mediterranean Sea, hosting a next generation underwater neutrino observatory. The infrastructure will host two neutrino detectors: ORCA, offshore Toulon (France) at a depth of $2500 \mathrm{~m}$, and ARCA, offshore Capo Passero (Italy) at a depth of $3500 \mathrm{~m}$. ORCA will be a Mton scale detector, optimised to detect neutrinos of tens of $\mathrm{GeV}$. It will be dedicated to study neutrino oscillations aiming in particular at determining the mass hierarchy. ARCA will be the km3-scale neutrino telescope in the Northern hemisphere, dedicated to the search for astrophysical neutrino sources in the TeV$\mathrm{PeV}$ range. Its location will allow surveying a large part of the sky, including most of the Galactic Plane and the Galactic Centre. The first detection units were deployed and the data analysis is ongoing to validate performances of both the detectors. In this paper we will give a general overview of the project, reporting its present status as of September 2019 and the future perspectives.
\end{abstract}

European Physical Society Conference on High Energy Physics - EPS-HEP2019 -

10-17 July, 2019

Ghent, Belgium

${ }^{*}$ Speaker. 


\section{Introduction}

The KM3NeT Collaboration is constructing a multi-site research infrastructure hosting a next generation underwater neutrino observatory. It will be distributed in two sites in the Mediterranean Sea with different detector configurations: ORCA (Mton scale 2500 depth offshore Toulon, France) and ARCA (Gton scale, $3500 \mathrm{~m}$ offshore Capo Passero, Italy). The ORCA detector is optimised to detect neutrinos of tens of $\mathrm{GeV}$ with the aim to study the neutrino oscillation physics and in particular to determine the mass hierarchy. ARCA is the high-energy component and is dedicated for the search of extraterrestrial neutrino sources in the TeV-PeV range. The main physics cases for KM3NeT/ARCA are the search for diffuse neutrino fluxes, in particular the investigation from a complementary field of view and with better angular resolution of the IceCube findings [1], and the search for point-like neutrino sources, with a particular focus on the galactic ones. The status as of September 2019 of KM3NeT and the future prospects of the project will be presented in this paper.

\section{The KM3NeT detector design}

The KM3NeT detectors are three dimensional arrays of optical sensors detecting the Cherenkov light induced in water by the secondary charged particles produced in neutrino interactions. The arrival times and the amount of the light collected by optical sensors and the positions of the latter are used to reconstruct the secondary particles. From these informations the neutrino arrival directions can be inferred and their energies estimated.

The basic unit of the detectors is the Digital Optical Module (DOM) [2]. The DOM is a 17inch diameter pressure-resistant glass sphere containing 31 3-inch photomultiplier tubes (PMTs) with their associated electronics for digitisation and data transmission as well as the calibration instrumentation. Nineteen of these PMTs are located in the lower hemisphere, and are thus looking downwards, while 12 in the upper hemisphere are looking upwards (Fig.1). This novel design offers significant improvements with respect to optical modules with a single large-area PMT: the total photocathode area is about three times larger, the field of view covers almost the full solid angle, pixelisation allows for high-purity photon counting and directional sensitivity.

A vertical sequence of 18 DOMs forms a Detection Unit (DU) [2]. Two thin parallel ropes hold the DOMs in place. The DU is anchored on the seabed and kept taut by a submerged buoy at its top. A vertical "backbone" cable, running along the full length of the DU, provides connectivity for power and data transmission. This cable is an oil-filled plastic tube, in equi-pressure with sea water, containing electrical wires and optical fibres, with a breakout box at each DOM. A sketch of the DU is shown in Fig.1.

A set of 115 DUs, arranged with a roughly circular footprint, forms a "building block". The DUs are connected to junction boxes and finally to shore by means of a main electro-optical cable. The architecture is intrinsically modular allowing for a staged implementation of the telescope.

\section{The ORCA detector}

The average ORCA detector is located at the KM3NeT-France site off-shore Toulon and at a depth of $2450 \mathrm{~m}$ (see Fig. 2). The DOM spacing along the DU is $9 \mathrm{~m}$ and the average distance 


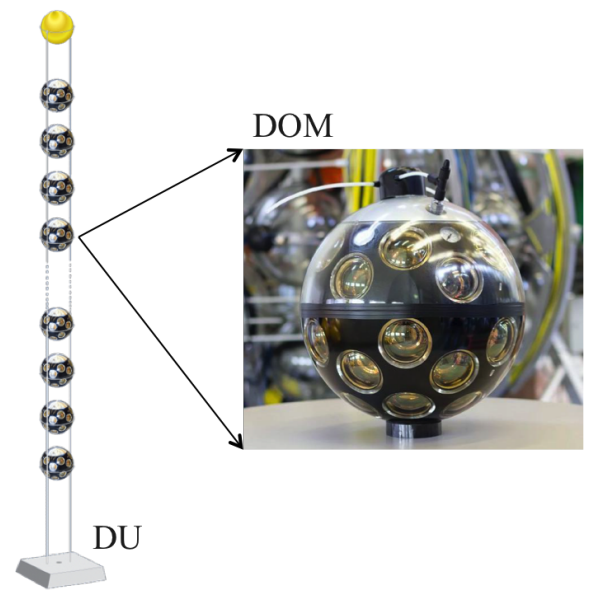

Figure 1: Schematic outline of the KM3NeT DU and a picture of the DOM.

among the DUs is $23 \mathrm{~m}$. ORCA will consists of one building block, as shown in Fig. 2, with an instrumented mass of 8 Mton.
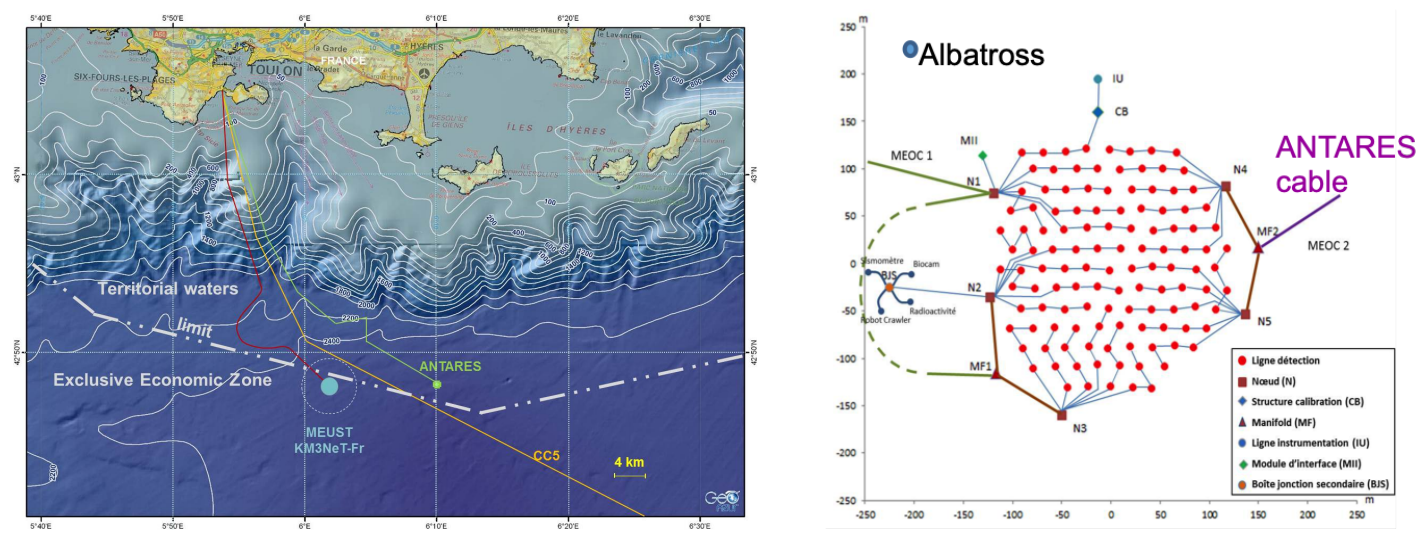

Figure 2: Left: map of the Mediterranean Sea south of Toulon, France. The location of the KM3NeTFrance and ANTARES installations are indicated. Right: layout of the ORCA detector, depicting the 115 DUs, cables and connection devices of the full array.

An angular resolution of $5^{\circ}$ and and energy resolution of $30 \%$ at $10 \mathrm{GeV}$, for both track and shower channels, are estimated for ORCA with Monte Carlo simulations. An algorithm based on the Random Decision Forest technique is used to perform particle identification. Monte Carlo simulations show that $90 \%$ of electron neutrinos and $70 \%$ of muon neutrinos are correctly identified at $10 \mathrm{GeV}$ [2]. The algorithm is also able to suppress atmospheric muon background and noise to $3 \%$ level, preserving $95 \%$ of the neutrino signal. With a Log-likelihood ratio test statistic is then possible to estimate the expected sensitivity to exclude the wrong hierarchy. The ORCA detector could be able to resolve the question of the neutrino mass ordering after only 3 years of data taking, as shown in Fig. 3 (left).

Once the hierarchy is established, it will be possible to measure the oscillation parameters such as the mixing between the second and third neutrino mass eigenstates, still the least well measured. 
ORCA will measure $\sin ^{2} \theta_{23}$ and $\Delta \mathrm{m}_{23}^{2}$ via the disappearance of $v_{\tau}$ in the atmosphere flux. The expected precision is shown in the right panel of Fig. 3.
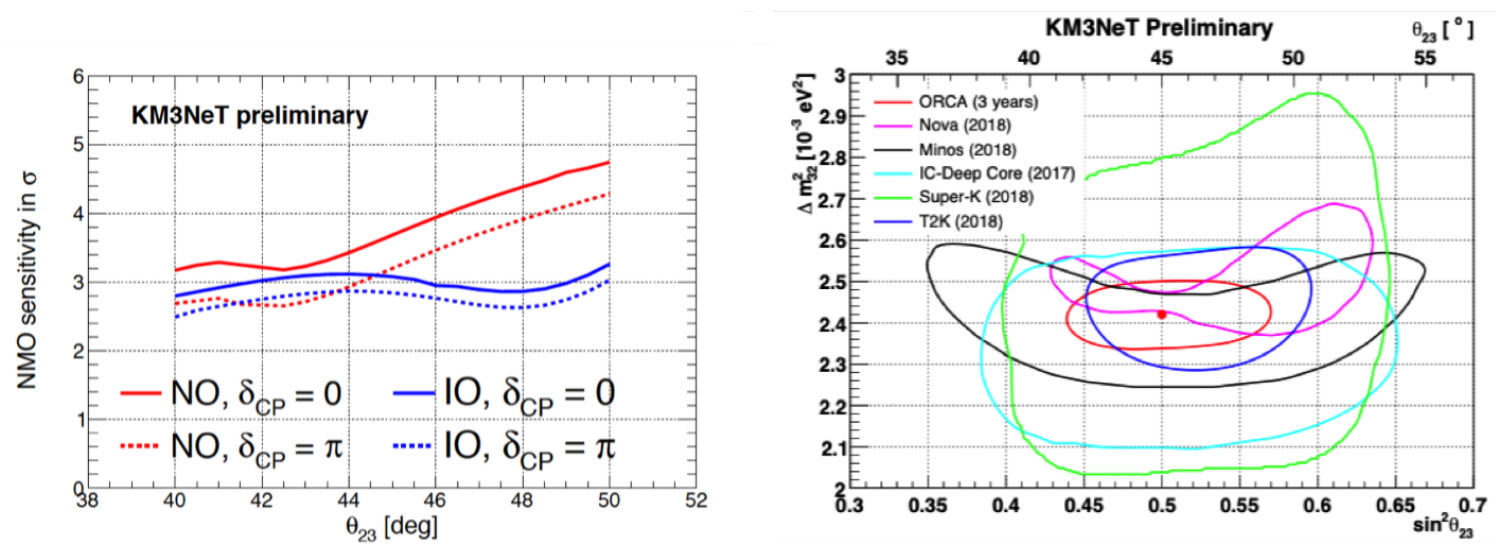

Figure 3: Left: detector sensitivity to reject the wrong hierarchy after 3 years of data taking. Right: expected precision for $\sin ^{2} \theta_{23}$ and $\Delta \mathrm{m}_{23}^{2}$, compared with other experiments.

The possibility to test the unitarity of the PMNS matrix is under study. For vertically up-going neutrinos, with a baseline of the Earth diameter, the $v_{\mu}$ disappearance in $v_{\tau}$ is maximum at about 24 $\mathrm{GeV}$, well above the detector energy threshold. A better than $10 \%$ precision on the $v_{\tau}$ appearance rate in one year (Fig. 4, left). It also will be possible to investigate the existence of sterile neutrinos. ORCA will be able to search also for sterile neutrinos. A preliminary estimate of the sensitivity in a $3+1$ scenario is shown in Fig. 4 (right) in the $\left|U_{\mu, 4}\right|^{2}-\left|U_{\tau, 4}\right|^{2}$.
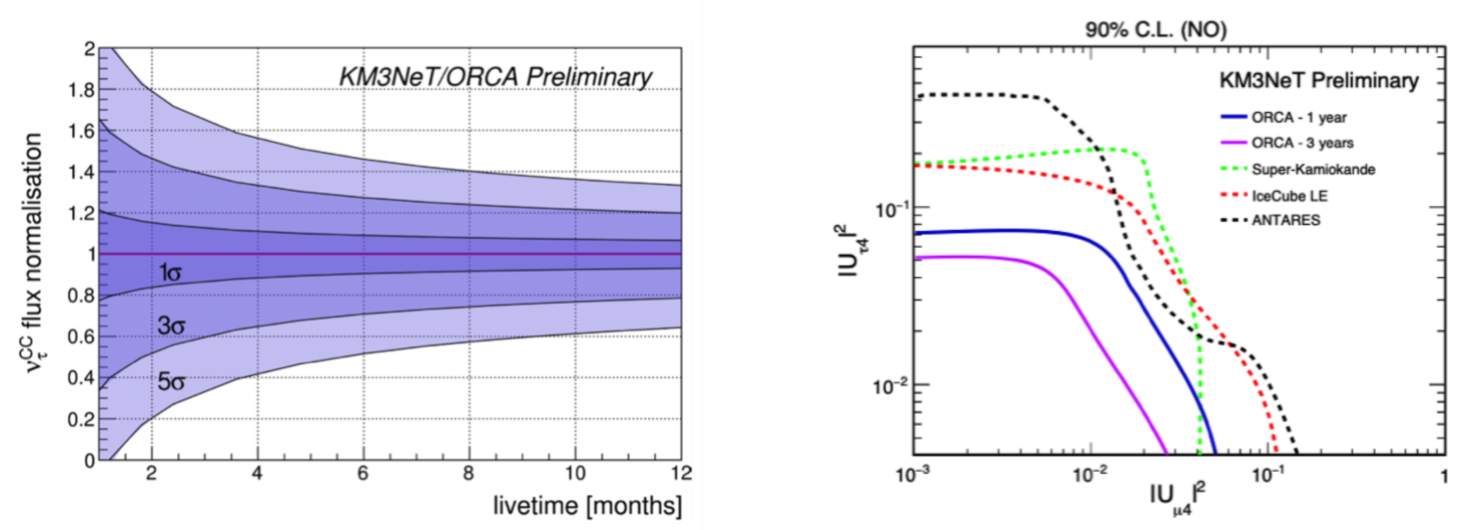

Figure 4: Left: precision on the rate of $v_{\tau}$ appearance as a function time. The true value 1 is assumed to be the Standard Model expectation. Right: preliminary ORCA sensitivity to sterile neutrinos.

\section{The ARCA detector}

The ARCA telescope is located at the KM3NeT-Italy site, about $100 \mathrm{~km}$ off-shore Portopalo di Capo Passero (Italy), at a depth of $3500 \mathrm{~m}$ (see Fig. 5). The vertical DOM spacing along the DU 

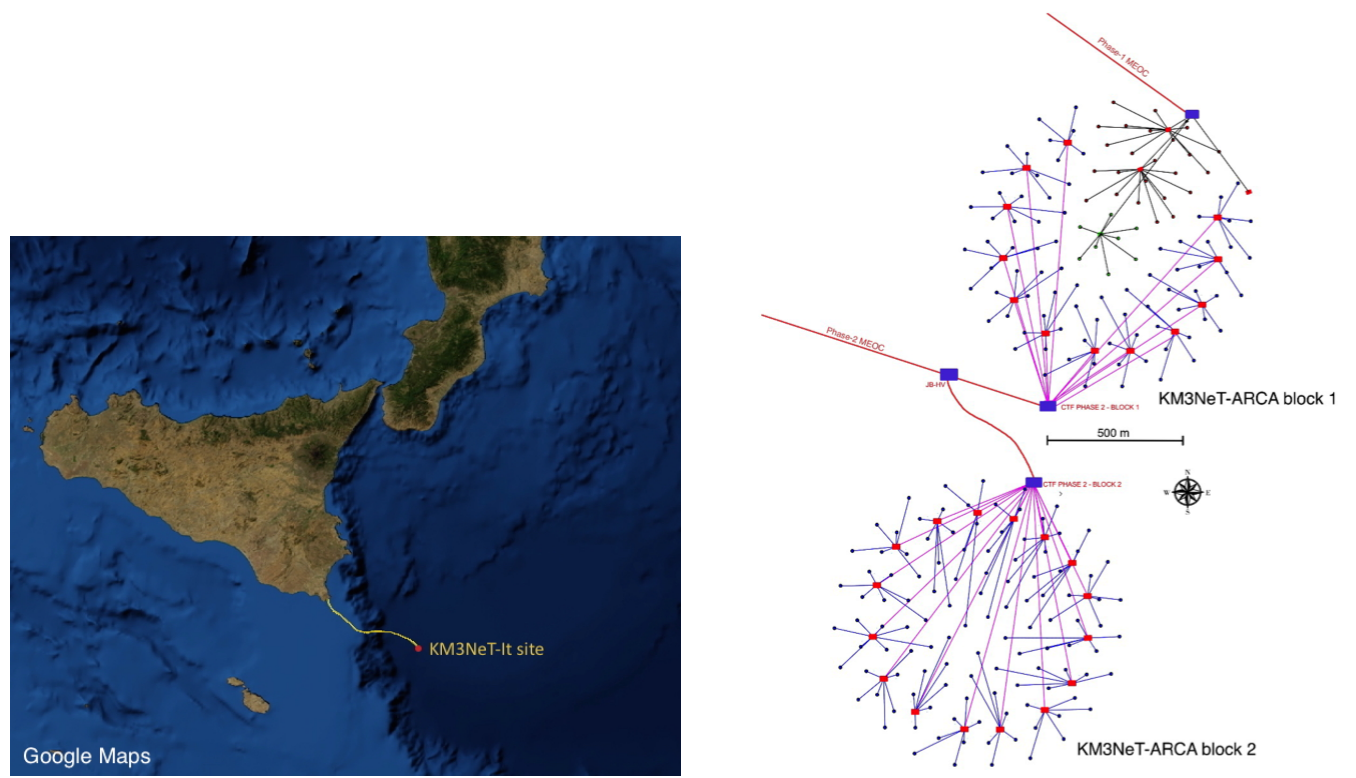

Figure 5: Left: map of the Mediterranean Sea south of Sicily, Italy. The location of the KM3NeT-Italy is indicated. Right: layout of the ARCA detector, depicting the two building blocks, cables and connection devices.

is $36 \mathrm{~m}$ with an average distance among DUs of $90 \mathrm{~m}$. ARCA will consists of two building blocks as shown in Fig. 5 and will have an instrumented volume of $1 \mathrm{~km}^{3}$.

The golden channel is the detection of long track muons produced in CC muon neutrino interactions. For this kind of events an angular resolution better than $0.1^{\circ}$ and an energy resolution of $30 \%$ in log-energy are expected for neutrino energies greater than $10 \mathrm{TeV}$. Shower events, induced by all flavour neutrino interaction in $\mathrm{NC}$ and electron neutrinos in $\mathrm{CC}$, are also detectable. Monte Carlo simulations show that showers events will be reconstructed with an angular resolution better than $2^{\circ}$ and energy resolution better $\approx 5 \%$ at $1 \sigma$ [2].

The astrophysical diffuse flux observed by IceCube can be considered as a guaranteed signal [3]. Its detection and detailed investigation with ARCA was studied, assuming that the IceCube signal originates from an isotropic, flavour-symmetric neutrino flux following a power law spectrum with a cut-off at a few PeV (see Fig. 6, left).

The identification of point-like sources is one of the main goals of the ARCA telescope, for which the sensitivity to a generic $E^{-2}$ neutrino flux is shown in the right panel of Fig. 6. Thanks to its geographical location, the telescope will allow for surveying a large part of the Galactic Plane, including the Galactic Centre. The sensitivity of the ARCA neutrino telescope to a selection of Galactic neutrino sources has been evaluated and reported in Fig. 7 (left). The SNR RXJ1713.73946 can be observed with a significance of $3 \sigma$ within 5.5 years. Stacking the two most intense SNR sources the RXJ1713.7-3946 and the Vela Jr. the $3 \sigma$ observation can be achieved in 2.5 years (Fig. 7, right) [6].

\section{Present status and first results}

The KM3NeT detectors are both currently under construction. The first two strings of ARCA 

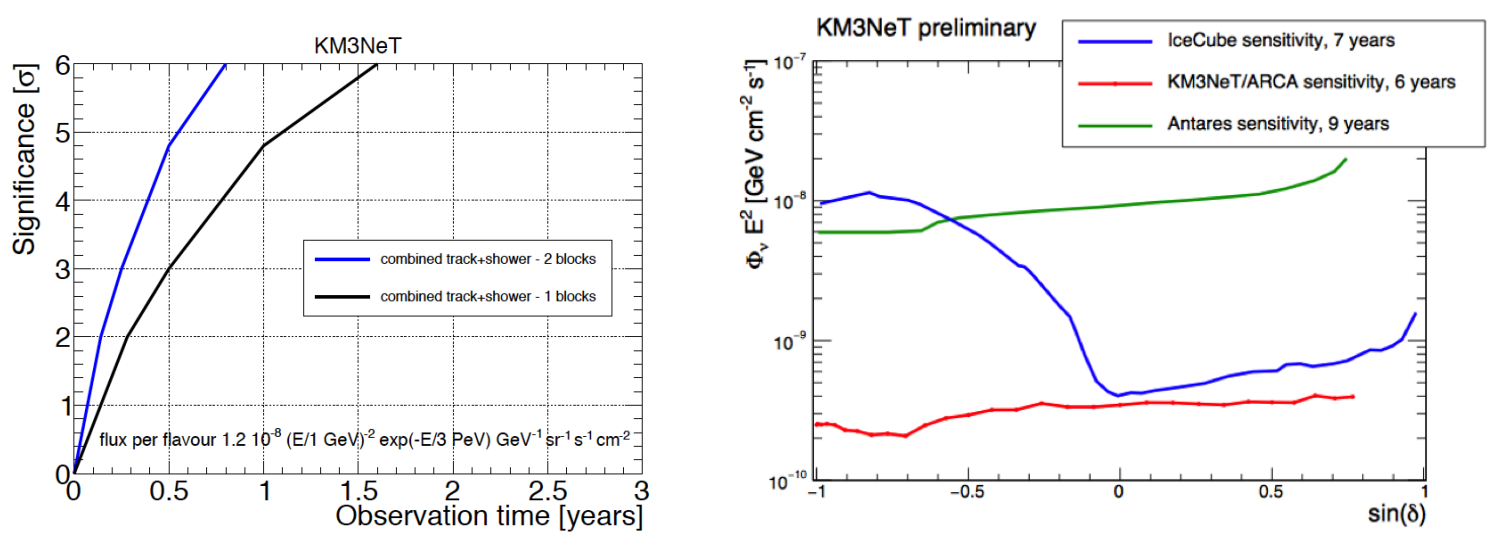

Figure 6: Left: Significance as a function of ARCA observation time for the detection of a diffuse flux of neutrinos corresponding to the signal reported by IceCube (see [2] for details). Right: ARCA sensitivity for a generic, unbroken $E^{-2}$ neutrino flux as a function of the source declination. For comparison, the corresponding IceCube [4] and ANTARES [5] results are also shown.
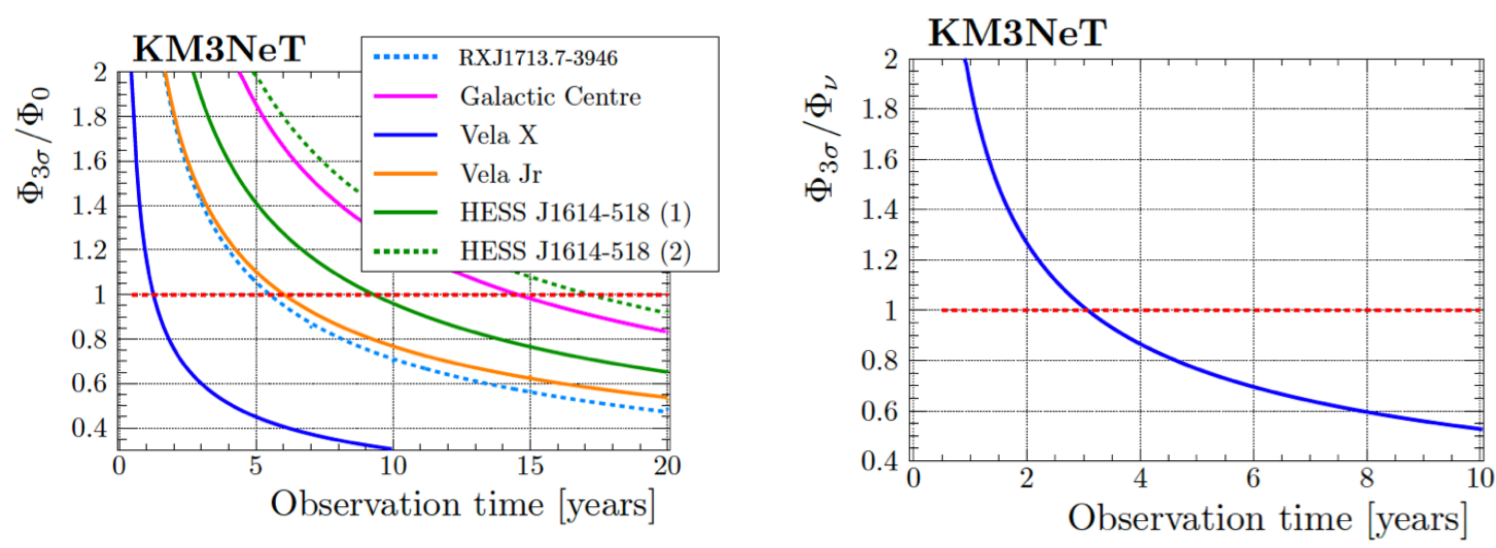

Figure 7: Right: ratio of ARCA sensitivity to the expectation flux as a function of the observation time for some galactic sources. See ref. [6] for details. Ratio of the discovery potential for ARCA to the expectation flux as a function of the observation time for the sources reported in the legend (right) and for the stacking analysis including RX J1713.7-3946 and Vela Jr (right).

were deployed in December 2015 and in May 2016. The first string of ORCA was deployed in September 2017 and 4 DUs were deployed during the 2019. The data presently collected with the 2 DUs of ARCA (ARCA2) and the first DU of ORCA (ORCA1) were reconstructed and analysed with the aim to select up-going neutrinos [7]. The reconstructed zenith angular distributions are shown in Fig. 8. The measured angular distribution is in good agreement with the Monte Carlo simulations. For ORCA1, 77 neutrino candidates are observed in the data sample, while 67 events are expected from atmospheric neutrino simulations and 4 events from atmospheric muon simulations. Simulations shown that the selected neutrino events have an average energy of a few tens of GeV. With ARCA2, the search for atmospheric neutrinos is more challenging due to the higher 
neutrino energy detection threshold and the smaller analysed livetime. For ARCA2, 6 up-going neutrino candidates are found in the analysed data sample while 2.5 events are expected from atmospheric neutrino simulations and 0 events from atmospheric muon simulations. The selected neutrino events show a typical energies of few hundreds of $\mathrm{GeV}$.
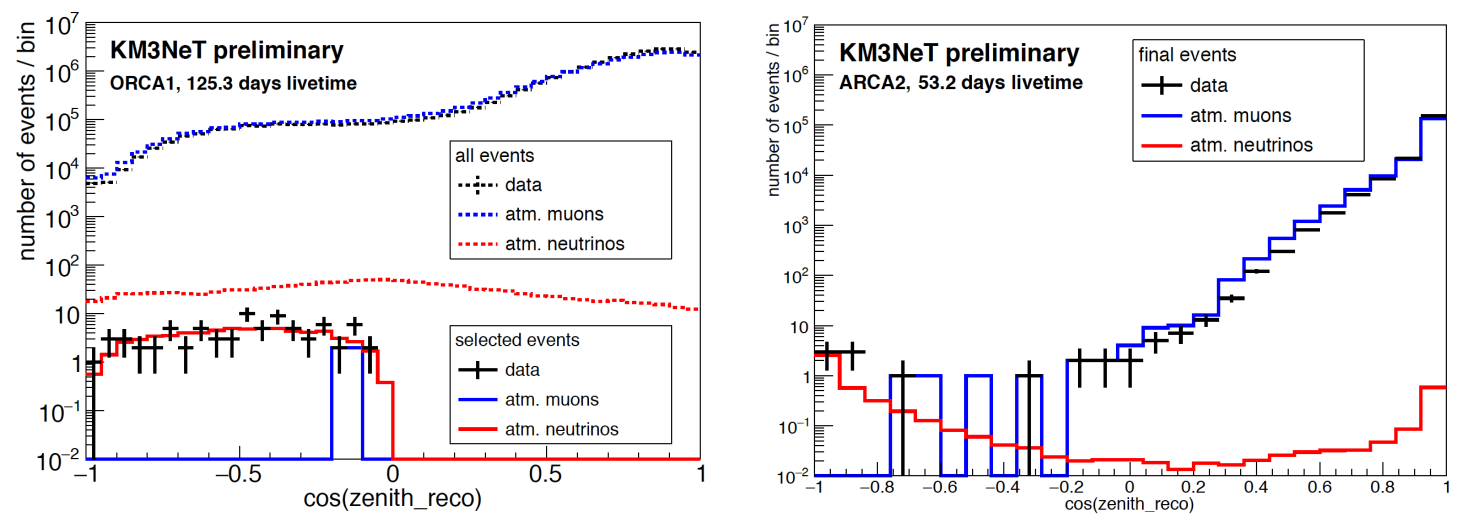

Figure 8: Reconstructed zenith distribution of ORCA1 events (left) and ARCA2 events (right).

\section{Conclusions}

The construction of the KM3NeT detectors has begun in the Mediterranean sea. The ORCA detector, devoted to study the atmospheric neutrino oscillations, will be able to determine the neutrino mass hierarchy in a few years of data taking. The ARCA telescope, with an instrumented volume of $1 \mathrm{~km}^{3}$, will offer independent confirmation of the IceCube flux and it will be optimised to study point-like astrophysical neutrino sources. In particular, it will profit of the advantage of its location to observe the Galactic Plane and the Galactic Centre.

The first two ARCA DUs and the first ORCA DU were deployed between 2015 and 2017 and data collected since their deployment are under analysis. Data are under analysis to reconstruct atmospheric muons and neutrinos in order to check the reconstruction algorithms and to optimise the event selection criteria for atmospheric muon rejection. First results show a good agreement with Monte Carlo simulations and then the functionality of the detectors.

\section{References}

[1] M. G. Aartsen et al. (IceCube Collaboration), European Physical Journal C79 (2019) 234.

[2] S. Adrián-Martínez et al. (KM3NeT Collaboration), J. Phys. G: Nucl. Part. Phys. 43 (2016) 084001.

[3] Aartsen M.G. et al. (IceCube Collaboration), Astrophys. J. 8333 (2016).

[4] M.G. Aartsen et al. (IceCube Collaboration), Astrophys. J. 835, 151 (2017).

[5] A. Albert et al. (ANTARES Collaboration), Phys. Rev. D 96, 082001 (2017)

[6] Aiello S. et al. (KM3NeT Collaboration), Astroparticle Physics 111100 (2019).

[7] R. Coniglione et al. for the KM3NeT Collaboration, PoS(ICRC2019)910. 